\title{
EXPORTSCORM: módulo SCORM para exportar objetos de aprendizagem do módulo Lição *
}

\author{
Marcela do Prado - Universidade Federal de Santa Maria - \\ marcela@unochapeco.edu.br \\ Felipe Martins Müller - Universidade Federal de Santa Maria - \\ felipe@inf.ufsm.br \\ André Zanki Cordenonsi - Universidade Federal de Santa Maria - \\ andrezc@inf.ufsm.br
}

Resumo. O avanço tecnológico proporciona uma nova realidade ao processo educacional, a possibilidade de usar ferramentas mais interativas no ensinoaprendizagem. Entre tantas ferramentas, os objetos de aprendizagem podem agir como facilitadores deste processo. Dispõe-se, no ambiente virtual de ensino-aprendizagem Moodle, de uma ferramenta, o módulo Lição, que permite combinar instrução e avaliação. Pode-se afirmar que se trata de um objeto de aprendizagem, considerando a baixa granularidade, no entanto, num contexto maior do que simplesmente uma sequência de conteúdos. O objetivo deste trabalho é apresentar um módulo, em conformidade com o padrão SCORM, para exportar os objetos de aprendizagem desenvolvidos no módulo Lição do Moodle, para firmar o conceito de reutilização de conteúdos nos ambientes virtuais de ensino-aprendizagem. Com isso, procura-se afirmar que as Lições do Moodle são objetos de aprendizagem, pois possuem as características de: reusabilidade, adaptabilidade, granularidade, acessibilidade, durabilidade $\mathrm{e}$ interoperabilidade.

Palavras-chave: Lição, Moodle, SCORM, Objetos de aprendizagem.

Abstract. The technological advance promotes a new reality to the education process, the possibility of using more interactive tools in teaching-learning. Among several tools, the learning objects may act like facilitators in this process. It is used, in on-line teaching-learning - Moodle, a tool, the Lesson module, which allows us to combine instruction and evaluation. It is possible to affirm that it is a learning object, considering the low granularity, however, in an environment bigger than simply a sequence of contents. Thus, this work aims to present a module, according to the pattern SCORM, in order to export learning objects developed in the Moodle Lesson Module, in order to firm the concept re-use of contents in on-line ambient of teaching-learning. This way, it is possible to affirm that the Moodle Lessons are learning objects, because they have characteristics, which are: re-use, adaptation, granularity, access, durability and interoperation.

Keywords: Lesson, Moodle, SCORM, Learning objects.

\section{Introdução}

O avanço tecnológico proporciona uma nova realidade ao processo educacional, a possibilidade de usar meios de comunicação mais interativos no ensino-aprendizagem o torna mais dinâmico, o que exige um estudante ativo na busca do conhecimento, engajado nas atividades educacionais suportadas através de um ambiente colaborativo de aprendizagem. Este pressupõe a construção, inserção e troca de informações pelos participantes num espaço de interação, visando à construção e à produção social do

" Artigo extraído da dissertação "EXPORTSCORM: módulo SCORM para exportar objetos de aprendizagem do módulo Lição" do Programa de Pós-Graduação de Informática da UFSM. 
conhecimento. Após conhecer o funcionamento deste espaço, ambiente virtual de aprendizagem, os estudantes estão preparados para interagir com as ferramentas disponíveis (MAGALHÃES, 2001).

Entre tantas ferramentas, os objetos de aprendizagem podem representar para o estudante um ganho de tempo no processo de aprendizagem, pois, podem ser facilitadores. Além disso, tornam as aulas mais interessantes e diversificadas.

O objeto de aprendizagem é uma ferramenta que possibilita o desenvolvimento de um recurso a ser usado tanto na educação presencial quanto a distância, suas características são: reusabilidade, autonomia, interatividade, granularidade, interoperabilidade e facilidade de busca.

Existem diversos conceitos sobre objetos de aprendizagem, mas aqui serão definidos como qualquer material didático desde que utilizado em um processo de ensinoaprendizagem com base tecnológica.

Um objeto de aprendizagem é construído para transmitir um conhecimento sobre uma matéria específica de maneira autônoma e rápida. Devido ao tempo empregado na sua elaboração, uma das principais características do objeto é que este deve ser reutilizável.

A adoção de um padrão para o desenvolvimento de objetos de aprendizagem visa a facilitar a portabilidade para diferentes ambientes de aprendizagem e contribui com a reutilização e interoperabilidade desses ambientes. Atualmente, existem diversas especificações que normatizam o desenvolvimento e a identificação dos objetos de aprendizagem, entre as principais pode-se destacar o ADL SCORM (Sharable Content Objetct Reference Model). O padrão SCORM, cujo foco é a preocupação na organização, sequenciamento e visualização do conteúdo, está fundamentado em algumas características como: reusabilidade, durabilidade, acessibilidade e interoperabilidade.

Dispõe-se, no ambiente virtual de ensino-aprendizagem (AVEA) Moodle, de diversos módulos que auxiliam no desenvolvimento de conteúdo para web, sendo que o mais completo é o módulo Lição. Esse permite combinar instrução e avaliação, oferecendo a flexibilidade de uma página web e a interatividade de um teste de conhecimento. Podese afirmar que se trata de um objeto de aprendizagem, levando em consideração a característica de granularidade, no entanto, num contexto maior do que simplesmente uma sequência de conteúdos.

Sabe-se que o Moodle possui um módulo SCORM/AICC que agrega um conjunto de conteúdos criados sob a forma de pacotes, o qual possibilita a importação, mas o que é desenvolvido em alguns módulos não pode ser exportado. A aderência do módulo Lição do Moodle aos padrões do padrão SCORM 2004 fundamenta o objetivo deste trabalho: a exportação dos objetos de aprendizagem desenvolvidos no módulo Lição do AVEA Moodle, para firmar a principal característica de reutilização de conteúdos e interoperabilidade. Ressalta-se que não há uma forma de reutilizar as Lições desenvolvidas no módulo em outros Learning Management System (LMS), pois, apesar do Moodle ser amplamente utilizado, não se preocupa com padrões para exportação. Segundo Silva (2010, p. 59), "o Moodle ainda não tem plugins ou módulos internos para a produção de Scorm".

Assim, pergunta-se: o módulo Lição pode ser considerado um objeto de aprendizagem? Ele pode ser reaproveitado em outros LMS?

O restante deste artigo está organizado da seguinte forma: a seção 2 discorre sobre o ambiente virtual de aprendizagem Moodle, objetos de aprendizagem e o módulo Lição. Na seção 3 é apresentado o desenvolvimento do módulo ExportSCORM. Para finalizar, as conclusões e as considerações finais são apresentadas na seção 4. 


\section{Ambiente Virtual de Ensino-Aprendizagem MOODLE}

O Moodle foi criado em 2001 pelo educador e cientista computacional Martin Dougiamas, com as vantagens de ser código fonte aberto, uma sólida filosofia educacional, a pedagogia socioconstrucionista, e uma comunidade ativa. Estas vantagens fazem do Moodle um dos Sistemas de Gestão do Curso (CMS - Course Management Systems) que visa a melhoria do ensino com proveito da Internet. A Universidade Aberta do Brasil (UAB) é uma das instituições que incentiva a utilização do Moodle nos seus cursos, isso "faz parte das políticas traçadas pelo MEC" (UAB, 2006).

O ambiente virtual de aprendizagem Moodle é um projeto livre que está sempre evoluindo para se adequar às necessidades da comunidade. O Moodle permite a gestão da aprendizagem e de trabalho colaborativo, permitindo a criação de cursos on-line, páginas de disciplinas, grupos de trabalho e comunidades de aprendizagem. Para isso, o Moodle tem ferramentas/módulos que auxiliam na administração do site, dos usuários, dos cursos e das atividades. Através da sua comunidade Moodle.org é possível agregar outras ferramentas/módulos desenvolvidas pelo Moodle ou por terceiros. A cada atualização, novidades e melhorias são incorporadas.

\subsection{Módulo Lição}

O módulo Lição do ambiente virtual de ensino-aprendizagem Moodle é a atividade/tarefa mais complexa e poderosa do AVEA - Moodle. Basicamente, uma lição é uma série de páginas web que apresenta informações e perguntas, uma combinação de instrução e avaliação de maneira on-line e partilhável, para outras disciplinas no AVEA. Permite incluir conteúdo em um curso de uma maneira flexível e interessante, como uma página web, a interatividade de um teste de conhecimento e capacidades de ramificação, caminhos alternativos, em que o estudante faz a seleção em cada página (RICE, 2008, p. 192).

Uma lição consiste num certo número de páginas web, normalmente, uma página da lição contém alguns materiais didáticos e uma questão e alternativas de resposta sobre o conteúdo. A questão é usada para testar a compreensão do estudante do conteúdo, dependendo da resposta escolhida, ele prossegue na lição ou retorna para a página de instrução ou para a página de reparação (RICE, 2008, p. 192-193). Esta sequência linear é a mais simples que o módulo Lição apresenta no Moodle, entretanto, necessita de planejamento da navegação, direcionando o mesmo para páginas específicas. O módulo proporciona outras sequências ou hierarquias complexas.

O módulo Lição tem algumas vantagens, como: integrar textos, imagens e vídeos através de páginas web, sem a necessidade de ser competente na criação ou editoração de documentos em HTML. Outra vantagem é a aplicação de exercícios, com caráter avaliativo complementar, ou seja, servem para avaliar o entendimento do conteúdo.

\subsection{Objetos de Aprendizagem}

Existem diversos conceitos sobre objetos de aprendizagem, no entanto, fazer o uso de maneira mais generalizada não limitará seu desenvolvimento, mas sim possibilitará que qualquer material seja considerado um objeto de aprendizagem. Basta utilizá-lo em um processo de ensino-aprendizagem com base tecnológica.

Wiley (2000) define objeto de aprendizagem como "[...] qualquer recurso digital que pode ser reusado para apoiar a aprendizagem. Esta definição inclui qualquer coisa que pode ser disponibilizada através da rede sob demanda, sendo isto grande ou pequeno."

O autor (2000b) ainda estabelece duas metáforas para os objetos de aprendizagem: uma associada ao brinquedo LEGO e a outra associada ao conceito do átomo. A metáfora 
que melhor define um objeto de aprendizagem é a do átomo, por mostrar que somente é agrupado se suas características forem compatíveis, na do LEGO essa ideia é vaga, pois os objetos combinam entre si desde que tenham o mesmo modelo.

Segundo Mendes, Souza e Caregnato (2004, p. 4-5) os objetos de aprendizagem são caracterizados por sete propriedades:

- reusabilidade: reutilizável diversas vezes em diversos ambientes de aprendizagem;

- adaptabilidade: adaptável a qualquer ambiente de ensino;

- granularidade: conteúdo em pedaços, para facilitar sua reusabilidade;

- acessibilidade: acessível facilmente via Internet para ser usado em diversos locais;

- durabilidade: possibilidade de continuar a ser usado, independente da mudança de tecnologia;

- interoperabilidade: habilidade de operar através de uma variedade de hardware, sistemas operacionais e browsers, intercâmbio efetivo entre diferentes sistemas.

No desenvolvimento de um objeto de aprendizagem deve-se levar em consideração o nível de agregação do objeto para aumentar seu reuso. Isto define o grau de granularidade, quanto maior o nível de granularidade, menor é sua reutilização, porém, é de fácil administração, quando o nível é baixo, sua reusabilidade é alta e de fácil localização, porém, intensificam-se as atividades para organizá-lo (WARPECHOWSKI, 2005).

No Brasil, há alguns projetos que desenvolvem objetos de aprendizagem como: o Programa Rede Internacional Virtual de Educação ${ }^{1}$ (RIVED), desenvolvido pelo Ministério da Educação (MEC), pela Secretaria de Educação a Distância (SEED), em parceria com a Secretaria de Ensino Médio e Tecnológico (SEMTEC); e a Coletânea de Entidades de Suporte ao uso de Tecnologia na Aprendizagem ${ }^{2}$ (CESTA) que sistematiza e organiza os objetos de aprendizagem desenvolvidos. O CESTA é um projeto desenvolvido pelo Centro Interdisciplinar de Novas Tecnologias na Educação (CINTED) da Universidade Federal do Rio Grande do Sul (UFRGS).

Outras instituições começaram a se interessar pela criação de material didático digital para potencializar o processo de ensino-aprendizagem. Nesse sentido, pode-se mencionar dois objetos de aprendizagem: o Laboratório Didático Virtual ${ }^{3}$ (LabVirt), uma iniciativa da Escola do Futuro da Universidade de São Paulo (USP), atualmente coordenado pela Faculdade de Educação; e o Mecânica Vetorial $^{4}$, um objeto de aprendizagem premiado pelo MEC.

Pode-se concluir, a partir da observação desses objetos de aprendizagem, que a Lição desenvolvida no módulo trata-se de um objeto de aprendizagem, desde que levada em consideração a granularidade, num contexto maior do que simplesmente uma sequência de conteúdos.

Ressalta-se a complexidade do módulo Lição, em que um professor pode desenvolver todo o conteúdo da disciplina a partir dele, no entanto, se por qualquer motivo, for necessário deixar de usar o Moodle, perde-se todo o trabalho, pois o módulo Lição é exclusivo deste AVEA, ou seja, ele não é aderente a nenhum padrão existente.

Por esse motivo, observam-se a seguir as características e como trabalhar com o modelo de referência SCORM, uma das especificações cujo foco é a preocupação na organização, sequenciamento e visualização do conteúdo, visando a elaboração do exportador para o módulo Lição.

\footnotetext{
${ }^{1}$ Disponível em: http://rived.mec.gov.br/

2 Disponível em: http://www.cinted.ufrgs.br/CESTA/

${ }^{3}$ Disponível em: http://www.labvirt.futuro.usp.br/

${ }^{4}$ Disponível em: http://www.mecanicavetorial.com/
} 


\section{MÓDULO PROPOSTO: EXPORTSCORM}

O Modelo de Referência para Objeto de Conteúdo Compartilhável (Sharable Content Object Reference Model - SCORM) é um conjunto unificado de especificações para a disponibilização de conteúdos e serviços de e-learning. Este conjunto de especificações define um modelo de agregação de conteúdo, um modelo de sequenciamento e um ambiente de execução para objetos de aprendizagem baseados na Web (ADL, 2010).

O padrão SCORM permite o empacotamento de conteúdo, que consiste em agregar todos os objetos de aprendizagem de uma unidade de aprendizagem em um único local, definidos a partir de uma determinada sequência e contendo os metadados deste conjunto. Ao final deste processo, através da 'scormização' do conteúdo, este se transforma em um arquivo único compactado denominado de pacote, o qual poderá ser importado para os ambientes virtuais de aprendizagem compatíveis, tal como o Moodle (RODRIGUES et al., 2009, p. 3).

Enfim, a utilização do padrão SCORM é adequada para ambientes computacionais que utilizam Web, o que permite, por exemplo, que um objeto de aprendizagem seja modificado facilmente e usado por diferentes ferramentas de desenvolvimento e plataformas e, também, ser aplicado em vários contextos. Com isso, é referência para a maioria das ferramentas de autoria, editores de texto e outros aplicativos para especificação de conteúdos para web, neste caso, os objetos de aprendizagem.

Para comprovar que as Lições desenvolvidas no módulo Lição do Moodle são objetos de aprendizagem necessita-se firmar o conceito de reutilizável em qualquer LMS. Por este motivo, será desenvolvido um módulo ExportSCORM.

O ExportSCORM ${ }^{5}$ é um módulo que pode ser agregrado ao Moodle, o qual exportará os objetos de aprendizagem desenvolvidos no módulo Lições do Moodle, em conformidade com o padrão SCORM.

Para desenvolvimento do módulo ExportSCORM, utilizou-se o modelo apresentado na Figura 2.

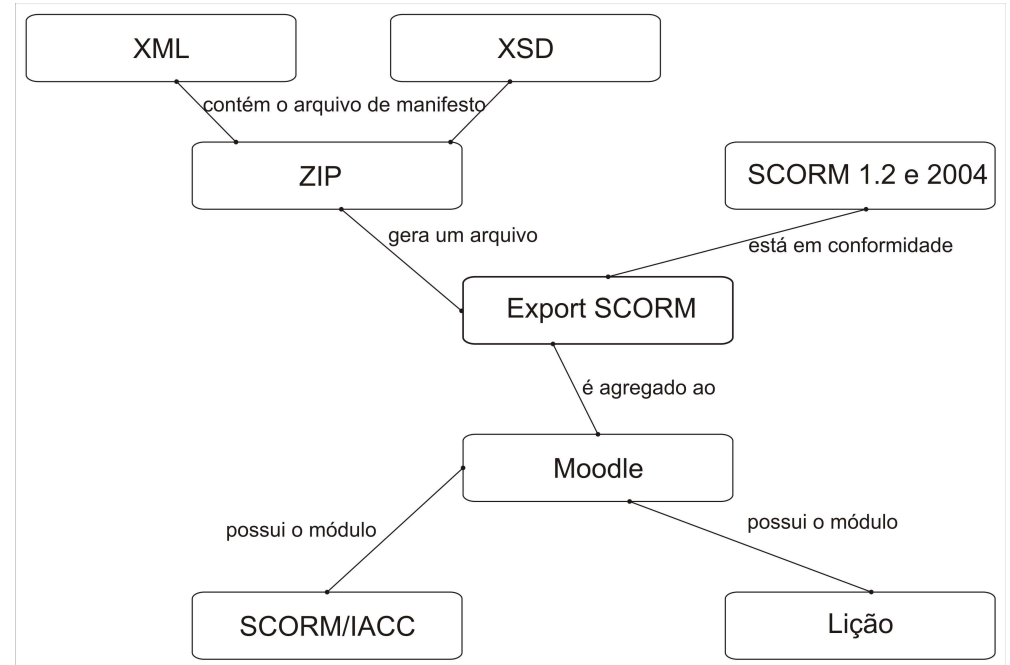

Figura 1 - Visão geral do módulo ExportSCORM

A Figura 1 apresenta uma visão geral do módulo ExportSCORM. O foco do módulo seria somente o SCORM 2004, no entanto, quando pesquisados players para a versão 2004, foram encontrados vários players pagos, por este motivo, decidiu-se por desenvolver também o módulo para o SCORM 1.2.

Esse módulo gera um pacote (conteúdo de embalagem), do tipo ZIP ${ }^{6}$, que contém o arquivo de manifesto, arquivo com as informações do objeto de aprendizagem e os

\footnotetext{
${ }^{5}$ Disponível em: http://moodle.org/mod/data/view.php?d=13\&rid=4713\&filter=1
} 
arquivos de configuração (Quadro 1), que são padrão do SCORM. Estes arquivos são compactados de acordo com o Modelo de Agregação de Conteúdo, o que torna o objeto de aprendizagem reutilizável nos LMS compatíveis com o SCORM.

Quadro 1 - Arquivos de configuração padrão do SCORM

\begin{tabular}{|l|l|}
\hline \multicolumn{2}{|c|}{ SCORM 2004 } \\
\hline adlcp_v1p3.xsd & elementos e/ou atributos que validam o conteúdo do pacote \\
\hline adlnav_v1p3.xsd & elementos e/ou atributos que validam a navegação das informações \\
\hline adlseq_v1p3.xsd & $\begin{array}{l}\text { elementos e/ou atributos que validam o sequenciamento das } \\
\text { informações. }\end{array}$ \\
\hline imscp_v1p1.xsd & elementos e/ou atributos que validam o conteúdo do pacote \\
\hline imsss_v1p0.xsd & $\begin{array}{l}\text { elementos e/ou atributos que validam o sequenciamento simples das } \\
\text { informações }\end{array}$ \\
\hline
\end{tabular}

Fonte: ADL (2009)

As informações sobre copyright, direitos de propriedade intelectual e de condições de uso não foram implementadas. Estas informações dependem da política de contratação e cessão dos direitos autorais de cada instituição.

No desenvolvimento dos arquivos de manifesto e das características do objeto, as informações se relacionaram com a base de dados do Moodle, como apresentado na Figura 2, em que não estão representados todos os campos das tabelas.
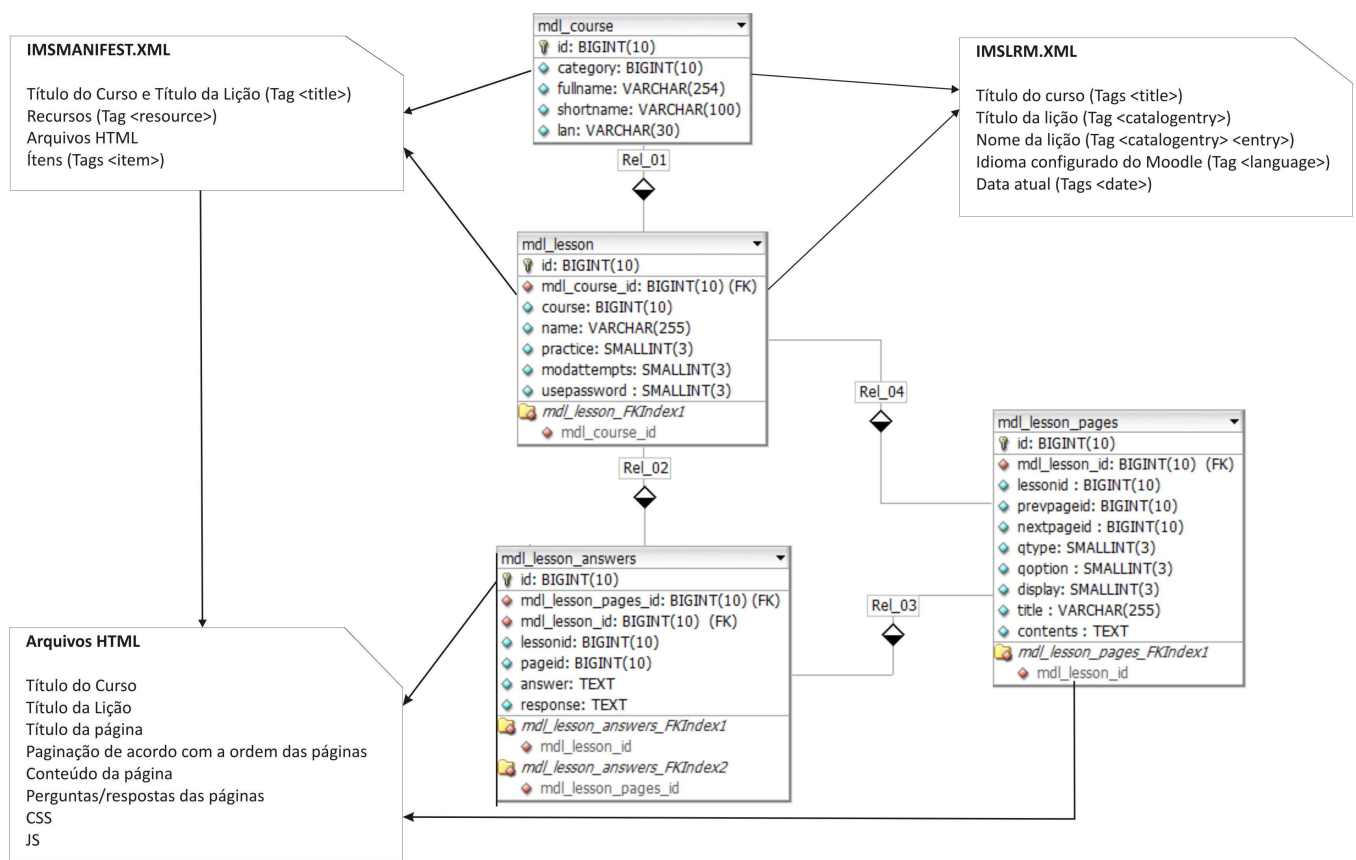

Figura 2 - Mapeamento do XML em relação ao banco de dados do Moodle 1.9.9

Foram utilizadas quatro tabelas para a criação dos arquivos HTML, imsmanifest.xml e imslrm.xml, que são:

- mdl_course - nome e código das disciplinas

- mdl_lesson - lições de cada disciplina

- mdl_lesson_answers - respostas e feedback das perguntas

- mdl_lesson_pages - título e conteúdo das páginas

\footnotetext{
${ }^{6}$ É um formato de compactação de arquivos.
}

V. $9 \mathrm{~N}^{\mathrm{o}} 1$, julho, 2011 
As tabelas mdl_course e mdl_lesson forneceram as informações para os arquivos imsmanifest.xml e imslrm.xml. E as tabelas $m d l \_l e s s o n \_a n s w e r s ~ e ~ m d l \_l e s s o n \_p a g e s$ fornecem as informações para a criação dos arquivos HTML, que fazem parte do imsmanifest.xml.

Com a instalação do ExportSCORM no módulo Lição foi adicionado a guia, como mostra a Figura 3.

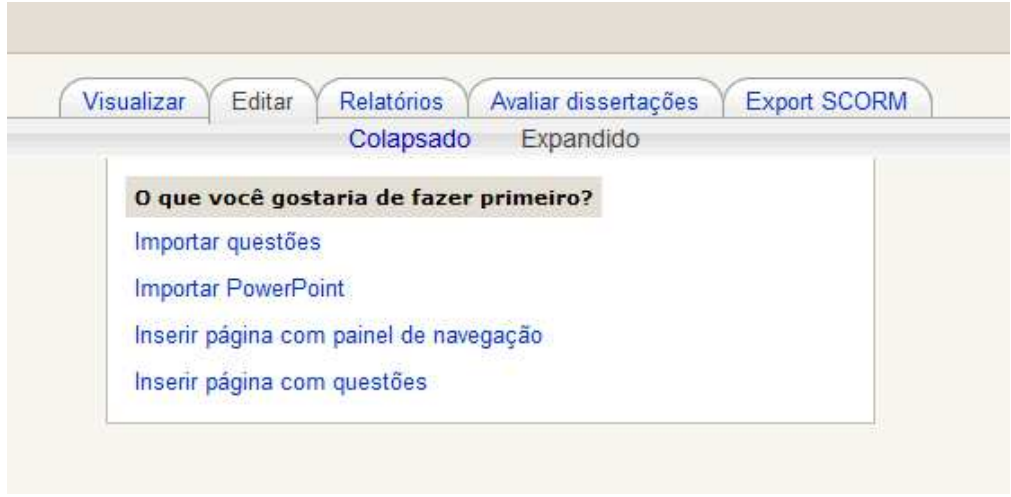

Figura 3 - Guia ExportSCORM

Nessa guia, depois de criado o objeto de aprendizagem no módulo Lição, ao editar a Lição o usuário poderá escolher o padrão de exportação, SCORM 1.2 ou SCORM 2004 (Figura 4).

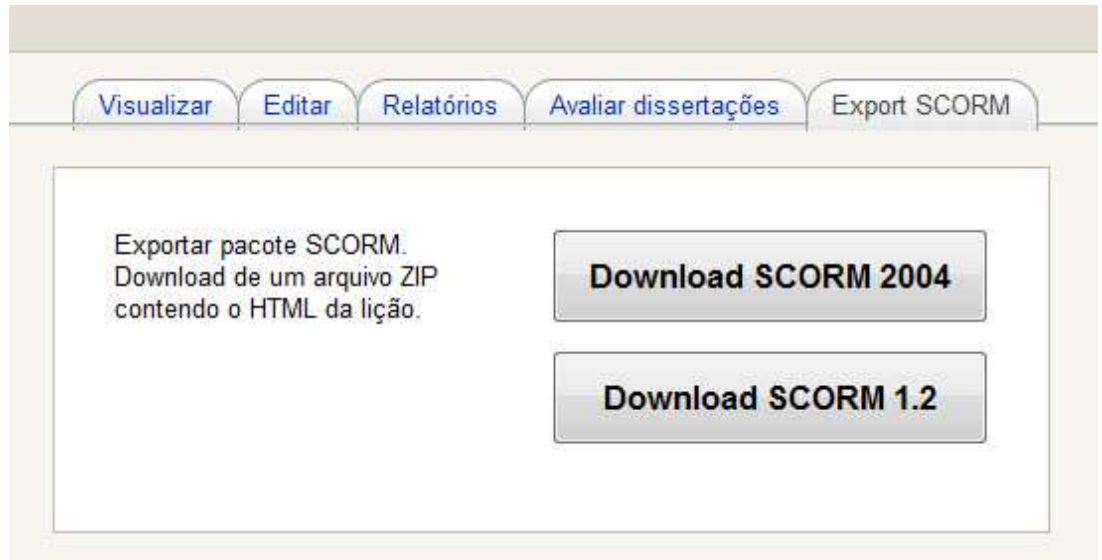

Figura 4 - Módulo ExportSCORM

Ao selecionar o padrão, será aberta uma janela para download do pacote de conteúdo do tipo ZIP. Após concluir o desenvolvimento do ExportSCORM utilizou-se do módulo SCORM/AICC do Moodle para importar o objeto de aprendizagem, que teve resultado satisfatório.

\section{CONCLUSÃO}

Hoje, a educação presencial e a distância possuem uma linha tênue que as distingue, a educação presencial incorpora algumas TIC e a educação a distância, a interação, para aprimorar o processo de ensino-aprendizagem. Nesse contexto, uma das TICs é incorporada e a interação é realizada através de plataformas de aprendizagem educacionais on-line ou ambientes virtuais de ensino-aprendizagem (AVEA). Os AVEA mais conhecidos são o Moodle, TelEduc, AulaNet, Tidia-Ae, WebCT, E-proinfo, Solar, ROODA, NAVI. Pode-se afirmar que o AVEA Moodle é um dos mais populares, sendo utilizado em 195 países. 
Outra TIC que vem se tornando uma alternativa aos professores no apoio ao processo de ensino-aprendizagem é o objeto de aprendizagem, Wiley (2000) define-o como um recurso digital reutilizável para apoiar a aprendizagem. Para o desenvolvimento de um objeto de aprendizagem, observou-se que a granularidade deve ser levada em consideração, pois, o nível de agregação do objeto tem relação com o seu reuso, quando o nível é baixo, sua reusabilidade é alta e de fácil localização.

O Moodle possui vários módulos, um deles desenvolve Lições, é uma atividade/tarefa mais complexa e poderosa do AVEA. Uma Lição é uma série de páginas web que apresenta informações e perguntas, uma combinação de instrução e avaliação de maneira online e partilhável, para outras disciplinas no AVEA (RICE, 2008, p. 192).

A complexidade do módulo Lição está relacionada com o desenvolvimento do conteúdo, sendo que um professor desenvolve a Lição para um determinado conteúdo, e que, se, por qualquer motivo, precisar deixar de usar o Moodle, perde todo o trabalho, pois o módulo Lição é exclusivo deste AVEA, ou seja, ele não é aderente a nenhum padrão existente.

Com a utilização do padrão SCORM no desenvolvimento de um objeto de aprendizagem, ou a scormização de um objeto de aprendizagem, é possível torná-lo reusável, acessível, interoperável e durável.

Segundo Silva (2010, p. 59), "o Moodle ainda não tem plugins ou módulos internos para a produção de Scorm”. Ressalta-se que não há uma forma de reutilizar as Lições desenvolvidas no módulo em outros LMS, pois, apesar do Moodle ser amplamente utilizado, não se preocupa com padrões para exportação. Por este motivo, desenvolveuse um módulo, ExportSCORM, para exportar as Lições desenvolvidas no Moodle, baseado no modelo de referência SCORM, para firmar a principal característica de um objeto de aprendizagem, a de reutilização de conteúdos e interoperabilidade. Esse módulo faz a "scormização" do objeto de aprendizagem desenvolvido no módulo Lição, que gera um pacote, do tipo ZIP, que contém o arquivo de manifesto, arquivo com as informações do objeto de aprendizagem e os arquivos de configuração, de acordo com o Modelo de Agregação de Conteúdo, o que torna o objeto de aprendizagem reutilizável nos LMS compatíveis com o SCORM.

A "scormização" foi validada no AVEA Moodle, e, também, no RELOAD e SCORM Player. No Moodle e no Reload Editor validou-se tanto o pacote do SCORM 1.2 quanto do SCORM 2004. No Reload SCORM 1.2 Player validou somente para o pacote do SCORM 1.2, e, no SCORM Player somente para pacote do SCORM 2004.

Nos AVEA TelEduc e Tidia-Ae não foi possível validar os pacotes de conteúdo gerados pelo módulo desenvolvido, ExportSCORM. O TelEduc não possui suporte ao SCORM e o Tidia-Ae informa que os padrões internacionais serão englobados no desenvolvimento, mas esta versão será disponibilizada somente em 2011 e, na versão de demonstração, não foi encontrada uma ferramenta que importasse o pacote de conteúdo SCORM. Nestes casos, tem-se a opção de disponibilizar o arquivo ZIP e utilizar-se de um dos players testados.

Com isso, afirma-se que a Lição desenvolvida no módulo é um objeto de aprendizagem, desde que levada em consideração a granularidade, num contexto maior do que simplesmente uma sequência de conteúdos, que pode ser reaproveitado nos AVEA, desde que disponham de player para SCORM. Neste caso, a Lição passa a ter todas as características de um objeto de aprendizagem, que são: reusabilidade, adaptabilidade, granularidade, acessibilidade, durabilidade e interoperabilidade. 


\section{REFERÊNCIAS}

ADL. Advanced Distributed Learning (ADL). Sharable Content Object Reference Model (SCORM®) 2004 4th Edition Content Aggregation Model (CAM), version 1.1, 2009.

Disponível

em: http://www.adlnet.gov/Technologies/scorm/SCORMSDocuments/2004\%204th\%20Edit ion/Documentation.aspx. Acesso em: 05 jun. 2010.

BRAGA, Cristiane Borges. DidaTIC: um ambiente digital de produção de conteúdo didático para a educação a distância. 116 f. 2008. Dissertação (Mestrado Integrado Profissional em Computação) - Universidade Estadual do Ceará, Centro de Ciências e Tecnologia. $\quad$ Fortaleza, Disponível em: http://www.mpcomp.pgcomp.uece.br/admin/arquivos/CristianeBraga2008.pdf. Acesso em: 25 jul. 2010.

COLE, Jason; FOSTER, Helen. Using MOODLE: teaching with the popular open. source course management system. 2. ed. Estados Unidos da América: O'Reilly Media, 2007.

MAGAlhãeS, Léo Pini (Org.). Projeto SAPIENS. fev. 2001. Disponível em: http://www.dca.fee.unicamp.br/projects/sapiens/Reports/rf2000/node29.html. Acesso em: 02 abr. 2010.

MENDES, Rozi Mara; SOUZA, Vanessa Inácio; CAREGNATO, Sônia Elisa. A propriedade intelectual na elaboração de objetos de aprendizagem. In: CINFORM Encontro Nacional de Ciência da Informação, 5, 2004. Disponível em: <http://dici.ibict.br/archive/00000578/01/propriedade_intelectual.pdf >. Acesso em: 18 mai. 2007.

MOODLE. Sobre o Moodle. out. 2009. Disponível em: http://docs.moodle.org/pt/Sobre_o_Moodle. Acesso em: 08 nov. 2010.

RICE, William H. Moodle 1.9 E-Learning Course Development: a complete guide to successful learning using Moodle 1.9. Birmingham: Packt Publishing, 2008.

RODRIGUES, Alessandra Pereira et al. Autoria e empacotamento de conteúdos. Revista Novas Tecnologias na Educação, Porto Alegre: UFRGS, Centro Interdisciplinar de Novas Tecnologias na Educação, v. 7, n. 3, dez. 2009. Disponível em: www.cinted.ufrgs.br/renote/dez2009/artigos/4b_alessandrapereira.pdf. Acesso em: 15 jul. 2010.

SILVA, Robson Santos da. Moodle para autores e tutores. São Paulo: Novatec, 2010.

STRUCHINNER, Mirian. Elementos Fundamentais para o Desenvolvimento de Ambientes Construtivistas de Aprendizagem a Distância. Rev. Tecnologia Educacional. Rio de Janeiro, ABT, v. 26, n. 142, jul./set. 1998.

UAB - Universidade Aberta do Brasil. ADI - Ambiente de Debate Iterativo. dez. 2006. Disponível em: http://mecsrv70.mec.gov.br/webuab/adi_orientacoes_1.php\#21. Acesso em: 20 jan. 2011. 
WARPECHOWSKI, Mariusa. Recuperação de Metadados de Objetos de Aprendizagem no AdaptWeb. 2005. 69 f. Dissertação (Mestrado em Ciência da Computação). Universidade Federal do Rio Grande do Sul. Porto Alegre, 2005. Disponível em: <http://www.inf.ufrgs.br/ erbd2005/Artigos/7924.pdf>. Acesso em: 18 mai. 2007.

WILEY, David A. Connecting learning objects to instructional design theory: a definition, a metaphor, and a taxonomy. In: The Instructional Use of Learning Objects, [online], 2000. Disponível em: http://reusability.org/read/chapters/wiley.doc. Acesso em: 12 jun. 2010.

YOKOYAMA, Bruno Pacheco; CARVALHO, Sérgio Teixeira de. Projeto e Implementação de Objetos de Aprendizagem SCORM. 8 f. 2007. Projeto Conclusão de Curso (Informática) - Universidade Federal de Goiás. Instituto de Informática, Goiás, $2007 . \quad$ Disponível em: http://www.assembla.com/spaces/pfclauro/documents/a7XKIShRur3RlbeJe5aVNr/dow nload/pfc-bruno.pdf. Acesso em: 01 ago. 2010. 